\title{
Contents
}

\section{Neutron Scattering for Biology}

T.A. Harroun, G.D. Wignall, J. Katsaras..................... 1

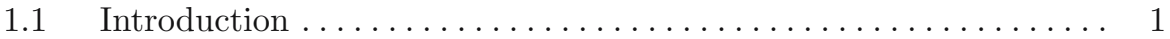

1.2 Production of Neutrons ........................... 2

1.3 Elements of Neutron Scattering Theory ................. 5

1.3.1 Properties of Neutrons ........................ 5

1.3.2 Energy and Momentum Transfer ................ 5

1.3.3 Diffraction ......................... 6

1.3.4 Scattering Length and Cross-Section .............. 7

1.3.5 Coherent and Incoherent Cross-Sections . . . . . . . . . . 8

1.4 Neutron Diffraction and Contrast..................... 10

1.4.1 Contrast and Structure ....................... 11

1.4.2 Contrast and Dynamics ....................... 13

1.4.3 Contrast and Biology ...................... 13

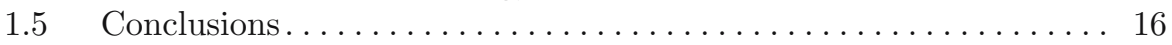

References ................................... 17

\section{Part I Elastic Techniques}

\section{Single Crystal Neutron Diffraction} and Protein Crystallography

C.C. Wilson, D.A. Myles ............................ 21

$2.1 \quad$ Introduction . . . . . . . . . . . . . . . . . . . . . 21

2.2 Single Crystal Neutron Diffractometers:

Basic Principles ........................... 22

2.2.1 Development of Single Crystal Neutron Diffractometers . . 25

2.2.2 Achievements of Neutron Macromolecular Crystallography at Reactor Sources................. 25

2.2.3 Developments at Spallation Sources ............... 28 
2.2.4 Forward Look for Instrumentation for Neutron Macromolecular Crystallography .......... . 29

2.2 .5 Improvements in Sources . . . . . . . . . . . . . . . 31

2.3 Information from Neutron Crystallography . . . . . . . . . . . . 32

2.3.1 Neutron Crystallography of Molecular Materials . . . . . . . 32

2.3.2 Neutron Crystallography in Structural Biology . . . . . . . . . 33

2.3.3 Sample and Data Requirements for Single Crystal Neutron Diffraction .............. 34

2.4 Brief Review of the Use of Neutron Diffraction

in the Study of Biological Structures . . . . . . . . . . . . . . 35

2.4.1 Location of Hydrogen Atoms . . . . . . . . . . . . . . . 36

2.4 .2 Solvent Structure . . . . . . . . . . . . . . . . . . . . . . . 38

2.4.3 Hydrogen Exchange . . . . . . . . . . . . . . . . . 39

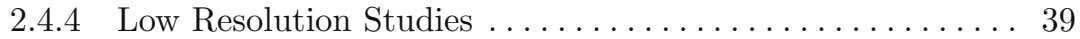

2.4.5 Other Biologically Relevant Molecules .............. 39

2.5 Recent Developments and Future Prospects . . . . . . . . . . . . 41

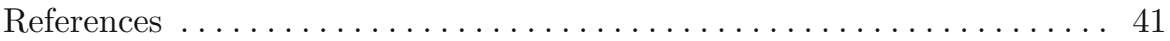

\section{Neutron Protein Crystallography:} Hydrogen and Hydration in Proteins

N. Niimura .................................. 43

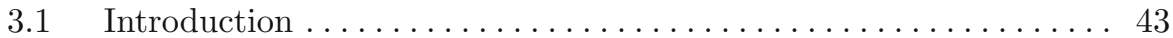

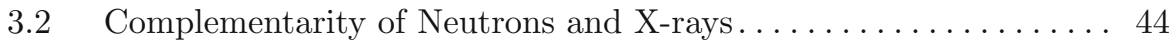

3.2.1 Refinement of Hydrogen Positions . . . . . . . . . . . . . . 44

3.2.2 Hydrogen Atoms Which Cannot be Predicted

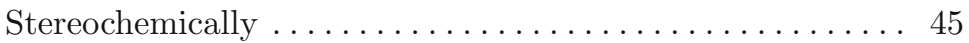

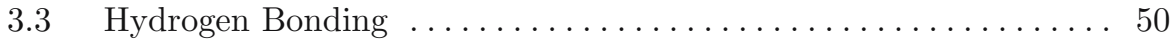

3.3.1 Weak and Strong Hydrogen Bonding .............. 50

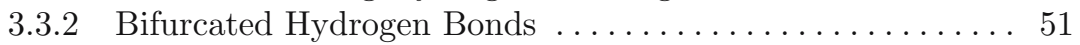

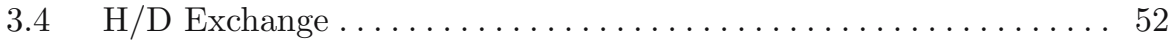

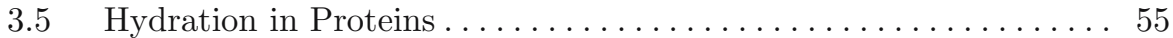

3.5.1 Experimental Observation of Hydration Molecules . . . . . . 55

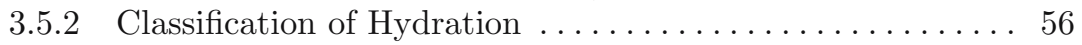

3.5.3 Dynamic Behavior of Hydration . . . . . . . . . . . . . . 58

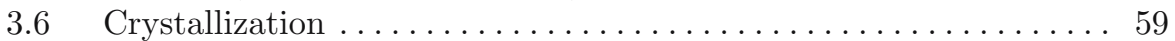

3.7 Conclusions and Future Prospects ................. 60

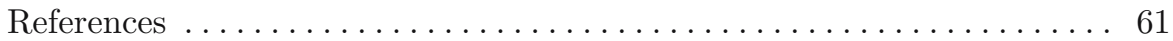

\section{Neutron Protein Crystallography:}

Technical Aspects and Some Case Studies

at Current Capabilities and Beyond

M. Blakeley, A.J.K. Gilboa, J. Habash, J.R. Helliwell, D. Myles,

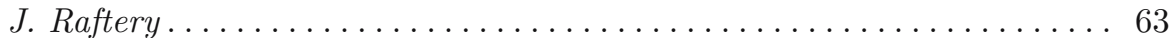

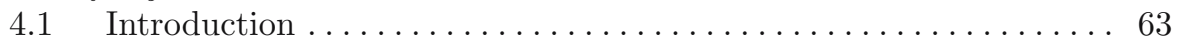

4.2 Data Collection Perspectives ....................... 64 
4.3 Realizing a Complete Structure:

The Complementary Roles of X-ray

and Neutron Protein Crystallography ................. 65

4.4 Cryo-Neutron Protein Crystallography ................ 66

4.5 Current Technique, Source,

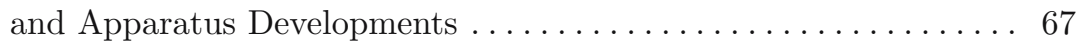

4.6 Plans for the $\mathrm{ESS}$ and $\mathrm{nPX} \ldots \ldots \ldots \ldots \ldots \ldots \ldots \ldots \ldots \ldots$

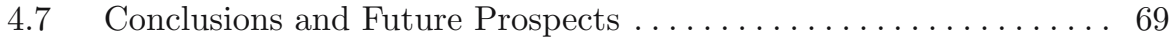

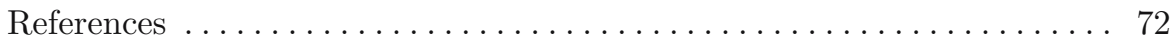

\section{Detergent Binding in Membrane Protein Crystals} by Neutron Crystallography

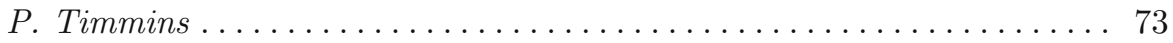

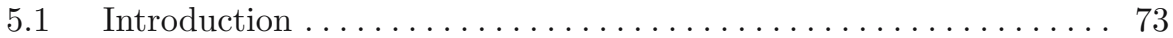

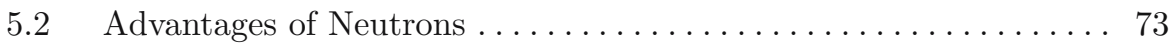

5.3 Instrumentation and Data Reduction $\ldots \ldots \ldots \ldots \ldots \ldots \ldots$

5.3.1 The Crystallographic Phase Problem ............. 76

5.4 Comparison of Protein Detergent Interactions

in Several Membrane Protein Crystals . . . . . . . . . . . 78

5.4.1 Reaction Centers and Light Harvesting Complexes ..... . 79

5.4 .2 Porins ............................ 80

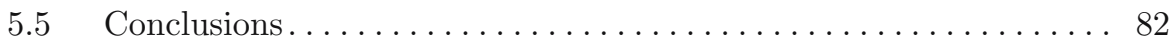

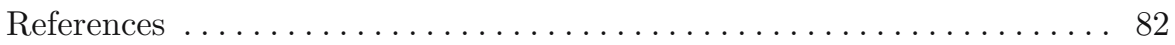

6 High-Angle Neutron Fiber Diffraction

in the Study of Biological Systems

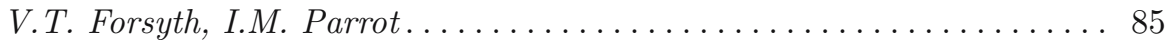

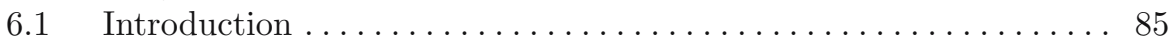

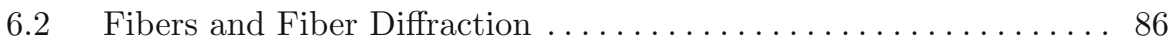

6.3 Neutron Fiber Diffraction: General Issues . . . . . . . . . . . . . . 87

$6.4 \quad$ Facilities for Neutron Fiber Diffraction . . . . . . . . . . . . . . . . . 90

$6.5 \quad$ Nucleic Acids . . . . . . . . . . . . . . . . . . . . . 92

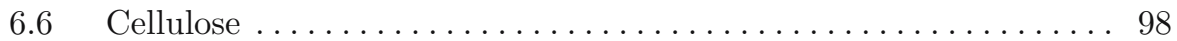

6.7 Conclusions and Future Prospects . . . . . . . . . . . . . . . 100

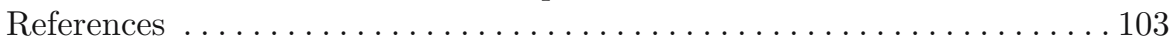

\section{Neutron Scattering from Biomaterials}

in Complex Sample Environments

J. Katsaras, T.A. Harroun, M.P. Nieh, M. Chakrapani, M.J. Watson,

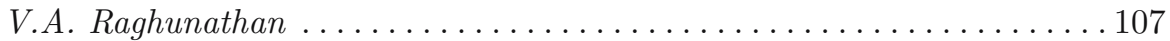

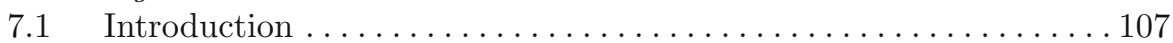

$7.2 \quad$ Alignment in a Magnetic Field . . . . . . . . . . . . . . . . . 107

7.2.1 Magnetic Alignment of Lipid Bilayers . . . . . . . . . . . . . 108

7.2.2 Neutron Scattering in a Magnetic Field: Other Examples . . 111

7.3 High Pressure Studies . . . . . . . . . . . . . . . . . . 113

7.3.1 Hydrostatic Pressure and Aligned Lipid Bilayers . . . . . . . 114 
7.3.2 High Pressure Neutron Scattering Experiments:

Other Examples ..................... 117

7.4 Shear Flow Induced Structures

in Biologically Relevant Materials . . . . . . . . . . . . . . . 118

7.4.1 Shear Cells Suitable for Neutron Scattering . . . . . . . . . . . . 118

7.4.2 Shear Studies of Biologically Relevant Systems . . . . . . . . . . 119

7.5 Comparison of a Neutron and X-ray Sample Environment . . . . . . 120

7.5.1 100\% Relative Humidity Sample Cells . . . . . . . . . . . . . 120

7.6 Conclusions . . . . . . . . . . . . . . . . . . . . . . . . . . . . . . 121

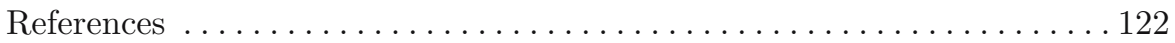

8 Small-Angle Neutron Scattering

from Biological Molecules

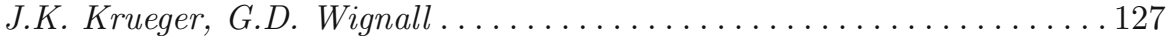

8.1 Introduction . . . . . . . . . . . . . . . . . . . . . . 127

8.1.1 Why Neutron Scattering is Appropriate and Comparison with Other Low- $Q$ Scattering Techniques . . . . . . . . . . 127

8.1.2 Complementary Aspects of Light, Small-Angle Neutron and X-ray Scattering for Solution Studies ........... 130

8.2 Elements of Neutron Scattering Theory . . . . . . . . . . . . . . 131

8.2.1 Coherent and Incoherent Cross-Sections . . . . . . . . . . . . . . 131

8.2 .2 Scattering Length Density . . . . . . . . . . . . . . . . . 134

8.2 .3 Contrast Variation ... . . . . . . . . . . . . . . . . . 135

8.3 Practical Aspects of SANS Experiments and Data Analysis . . . . . 137

8.3.1 SANS Instrumentation . . . . . . . . . . . . . . . . . . 137

8.3.2 The Importance of Absolute Calibration

and Having Well-Characterized Samples. . . . . . . . . . . . . 140

8.3 .3 Instrumental Resolution. . . . . . . . . . . . . . . . . . . . 142

8.3.4 Other Experimental Considerations and Potential Artifacts........................ 145

8.3.5 Data Analysis: Extracting Structural and Shape

Parameters from SANS Data and $P(r)$ Analysis........ 146

8.4 SANS Application:

Investigating Conformational Changes

of Myosin Light Chain Kinase. . . . . . . . . . . . . . . . . . . . . . . . 149

8.4.1 Solvent Matching of a Specifically Deuterated CaM

Bound to a Short Peptide Sequence . . . . . . . . . . . . . . 149

8.4.2 Contrast Variation of Deuterated CaM

Bound to MLCK Enzyme . . . . . . . . . . . . . 150

8.4.3 Mechanism of the CaM-Activation Step:

SAXS/SANS Studies of a (Deuterated) Mutant CAM . . . . 153

8.5 Conclusions and Outlook ......................... 155

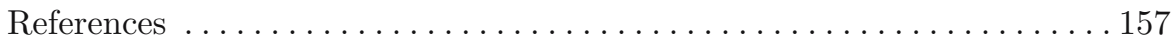


9 Small Angle Neutron Scattering

from Proteins, Nucleic Acids, and Viruses

S. Krueger, U.A. Perez-Salas, S.K. Gregurick, D. Kuzmanovic......... 161

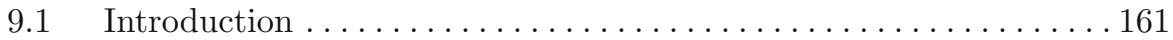

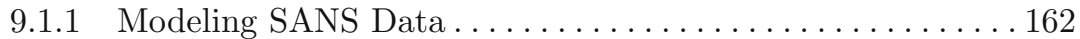

9.1 .2 Contrast Variation ........................... 164

9.1.3 Experimental Examples ........................ 165

$9.2 \quad$ Nucleic Acids: RNA Folding . ......................... 165

9.2.1 Compaction of a Bacterial Group I Ribozyme .......... 165

9.2.2 RNA Compaction and Helical Assembly.............. 170

9.3 Protein Complexes:

Multisubunit Proteins and Viruses..................... 172

9.3.1 Conformation of a Polypeptide Substrate

in Model GroEL/GroES Chaperonin Complexes.......... 172

9.3.2 Spatial Distribution and Molecular Weight of the Protein and RNA Components of Bacteriophage MS2 ........ 178

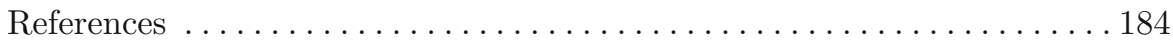

10 Structure and Kinetics of Proteins Observed

by Small Angle Neutron Scattering

M.W. Roessle, R.P. May.............................. 187

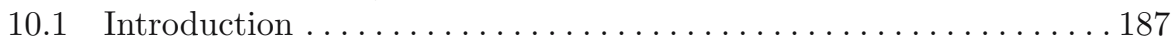

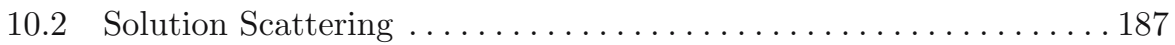

10.2.1 Specific Aspects of Neutron Scattering .............. 189

10.3 Time-Resolved Experiments: Dynamics vs. Steady State . . . . . . . 189

10.3.1 Protein Motions and Kinetics .................... 190

10.3.2 Cooperative Control of Protein Activity ............... 191

10.4 Protein Kinetic Analysis

by Neutron Scattering Experiments ..................... 192

10.4.1 Trapping of Reaction Intermediates:

The $(\alpha \beta)$-Thermosome ....................... 193

10.4.2 Quasi-static Analysis of Reaction Kinetics-The

Symmetric GroES-GroEL-GroES Complex . . . . . . . . . 196

10.4.3 Chasing Experiments (Slow Kinetics) . . . . . . . . . . . 199

10.4.4 Time Resolved Small-Angle Neutron Scattering _....... 200

10.5 Conclusions and Outlook ........................ 203

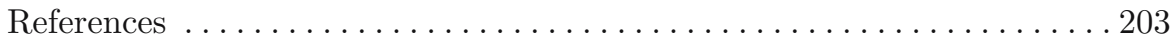

\section{Complex Biological Structures:}

\section{Collagen and Bone}

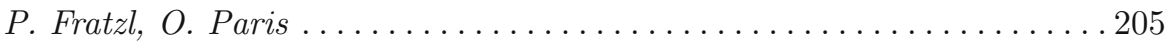

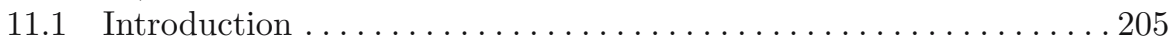

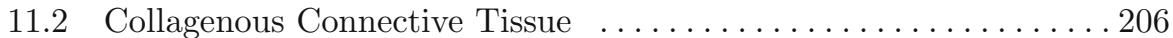

11.2.1 Structure and Dynamics by Neutron Scattering ......... 206 
11.2.2 Elastic and Visco-elastic Behavior of Collagen from In situ Mechanical Experiments

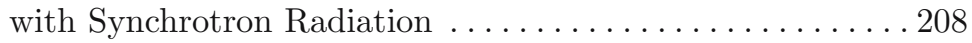

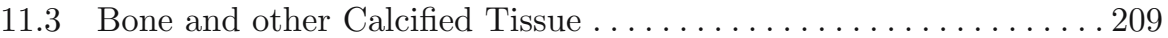

11.3.1 Structure of Mineralized Collagen - Contributions from Neutron Scattering . . . . . . . . . . . . . . . . . 209

11.3.2 Investigating the Hierarchical Structure of Bone. . . . . . . . 212 References ....................................221

\section{Structural Investigations of Membranes} in Biology by Neutron Reflectometry

C.F. Majkrzak, N.F. Berk, S. Krueger, U.A. Perez-Salas ........... 225

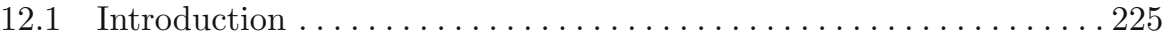

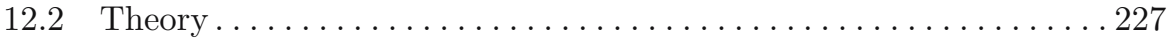

12.2.1 The Exact ("Dynamical") Solution . . . . . . . . . . . . . 227

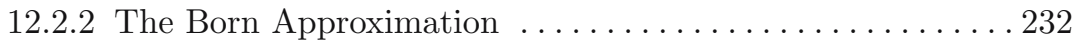

12.2 .3 Multilayers . . . . . . . . . . . . . . . . . . . . 233

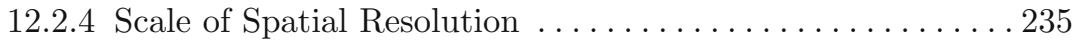

12.3 Basic Experimental Methods. . . . . . . . . . . . . . . . 236

12.3.1 Instrumental Configuration . . . . . . . . . . . . . . . 2237

12.3.2 Instrumental Resolution and the Intrinsic Coherence Lengths of the Neutron . . . . . 239

12.3.3 In-Plane Averaging . . . . . . . . . . . . . . . . . . 243

12.3.4 Q-Resolution for Specular Reflectivity, Assuming an Incoherent Beam ... . . . . . . . . . . . . . . . . . . 244

12.3.5 Measurement of the Reflectivity . . . . . . . . . . . . . . 246

12.3.6 Sample Cell Designs . . . . . . . . . . . . . . . . . . . 248

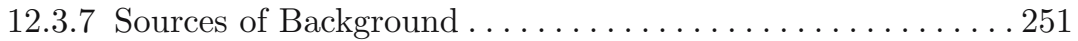

12.3.8 Multilayer Samples: Secondary Extinction and Mosaic . . . . 254

12.3.9 Data Collection Strategies for Time-Dependent Phenomena . . . . . . . . . . . . . . 254

12.4 Phase Determination Techniques . . . . . . . . . . . . . . . 255

12.4.1 Reference Films . . . . . . . . . . . . . . . . . . . . . 255

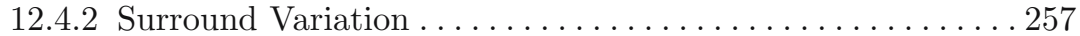

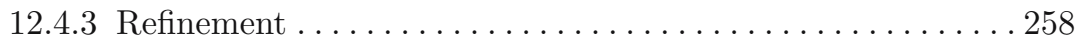

12.5 An Illustrative Example . . . . . . . . . . . . . . . . . . 259

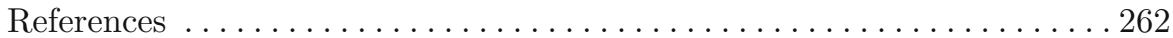

\section{Protein Adsorption and Interactions at Interfaces}

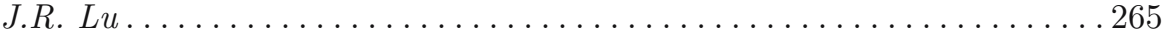

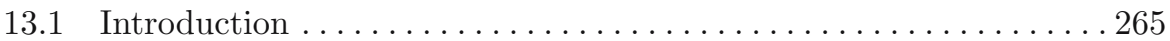

13.2 Neutron Reflection and Concept

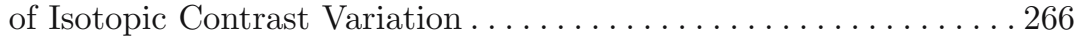

13.3 Adsorption of Other Proteins at the Air-Water Interface . . . . . . . 270 
13.4 Adsorption at the Solid-Water Interface: The Effect of Surface

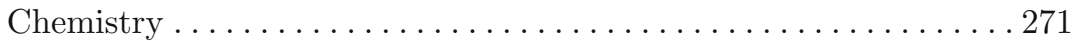

13.5 Interaction Between Surfactant and Protein . . . . . . . . . . . 277

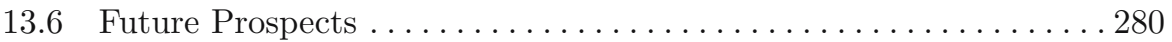

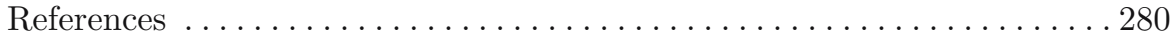

14 Complex Biomimetic Structures at Fluid Surfaces and Solid-Liquid Interfaces

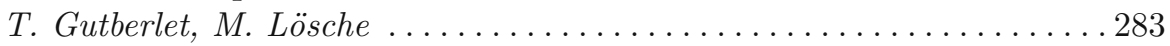

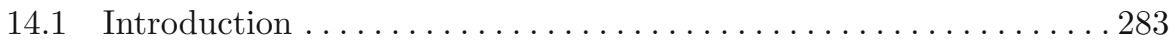

14.2 Surface-Sensitive Scattering . . . . . . . . . . . . . . . . . . . . . 284

14.2.1 Specular Reflectivity . . . . . . . . . . . . . . . . . . 284

14.2.2 Structure-Based Model Refinement . . . . . . . . . . . . . . 287

14.3 Floating Lipid Monolayers: Structural Investigations and the

Interaction of Peptides and Proteins with Lipid Interfaces . . . . . . 289

14.3.1 Single Phospholipid LMs . . . . . . . . . . . . . . . . . . 290

14.3.2 Functionalized Phospholipid LMs ............... 291

14.4 Lipopolymers . . . . . . . . . . . . . . . . . . . . . . . 292

14.5 Protein Adsorption and Stability

at Functionalized Solid Interfaces . . . . . . . . . . . . . . . . . . . 294

14.5.1 Hydrophobic Modified Interfaces . . . . . . . . . . . . . . . . . . 294

14.5.2 Hydrophilic Modified Interfaces . . . . . . . . . . . . . . . 296

14.6 Functionalized Lipid Interfaces

and Supported Lipid Bilayers . . . . . . . . . . . . . . . . . 297

14.6.1 Solid-Supported Phospholipid Bilayers . . . . . . . . . . . 297

14.6.2 Hybrid Bilayer Membranes . . . . . . . . . . . . . . . . . . . 299

14.6.3 Polymer-Supported Phospholipid Bilayers . . . . . . . . . . 301

14.7 Conclusions. . . . . . . . . . . . . . . . . . . . . . . . . . . . . 302

References ................................... 302

\section{Part II Inelastic Techniques}

15 Quasielastic Neutron Scattering in Biology, Part I:

\section{Methods}

R.E. Lechner, S. Longeville ............................ 309

15.1 Introduction . . . . . . . . . . . . . . . . . . . . . . . . . . . 309

15.2 Basic Theory of Neutron Scattering . . . . . . . . . . . . . . . 311

15.2.1 Van Hove Scattering Functions

and Correlation Functions . . . . . . . . . . . . . . . . . 313

15.2.2 The Elastic Incoherent Structure Factor . . . . . . . . . . . 316

15.2.3 Experimental Energy Resolution ................. 319

15.3 Instruments for QENS Spectroscopy in $(\boldsymbol{Q}, \omega)$-Space . . . . . . . . 323

15.3.1 XTL-TOF Spectrometers . . . . . . . . . . . . . . . . 323

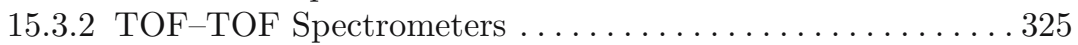




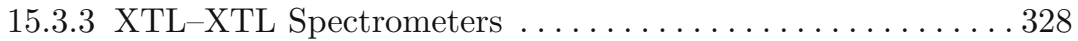

15.3.4 TOF-XTL Spectrometers .................... 333

15.4 Instruments for QENS Spectroscopy in $(\boldsymbol{Q}, t)$-Space . . . . . . . 335

15.4.1 NSE Spectrometers . . . . . . . . . . . . . . . . . . . 335

Spin $1 / 2$ and Larmor Precession $\ldots \ldots \ldots \ldots \ldots \ldots \ldots$

The Neutron Spin-Echo Principle . . . . . . . . . . . 337

Transmission of Polarizers and Analyzers . . . . . . . . . 339

Getting a Spin-Echo, as a Measure of the Polarization . . . . 340

Measuring Quasielastic Neutron Scattering . . . . . . . . . . 342

15.4.2 Neutron Resonance Spin-Echo Spectrometry . . . . . . . . . . . 344

15.4.3 Observation Function, Effect of Wavelength Distribution

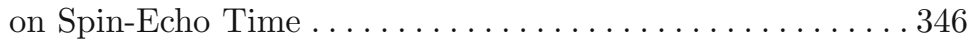

15.5 Miscellaneous Technical Points:

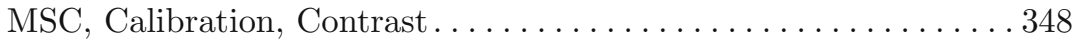

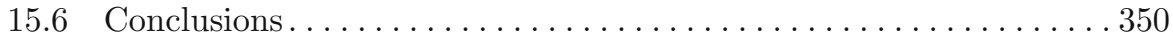

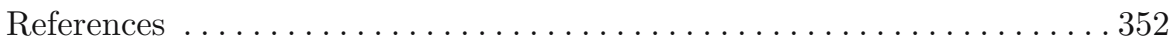

16 Quasielastic Neutron Scattering in Biology, Part II: Applications

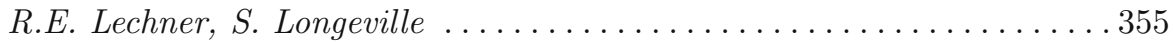

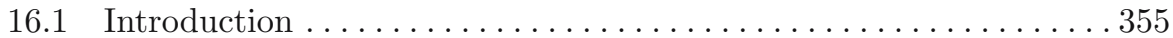

16.2 Dynamical Models . . . . . . . . . . . . . . . . . . . 356

16.2.1 Dynamical-Independence Approximation . . . . . . . . . . . . 356

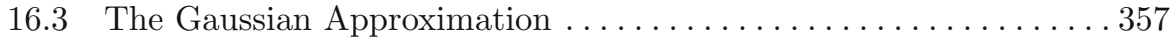

16.3.1 Simple Translational Diffusion . . . . . . . . . . . . . . 358

16.3.2 Three-Dimensional Diffusion of Protein Molecules in Solution (Crowded Media) . . . . . . . . . . . . . 359

16.3.3 Vibrational Motions, Phonon-Expansion and Debye-Waller factor (DWF), Dynamic Susceptibility . . . . 361

16.3.4 Vibrational Density of States of the Light-Harvesting Complex II of Green Plants . . . . . 364

16.4 Non-Gaussian Motion . . . . . . . . . . . . . . . . . . . . 367

16.4.1 Atomic Jump Motions Described by Rate Equations . . . . . . 368

16.4.2 Confined or Localized Diffusive Atomic and Molecular

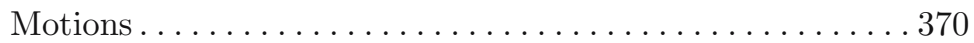

16.4.3 Environment-Dependence of Confined Diffusive Protein Motions: Example Lysozyme . . . . . . . . . . . . . . . . . . . . 371

16.4.4 Change of Protein Dynamics on Ligand Binding: Example Dihydrofolate Reductase . . . . . . . . . . . 374

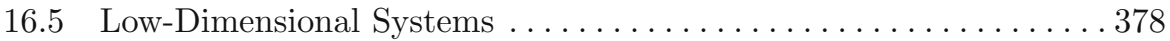

16.5.1 Two-Dimensional Long-Range Diffusion of Rotating Molecules....................... 378

16.5.2 Dynamical Transition and Temperature-Dependent Hydration: Example Purple Membrane.............. 383 


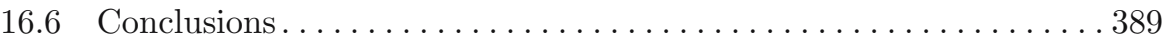

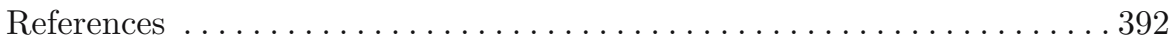

\section{Conformational Dynamics Measured}

\section{with Proteins in Solution}

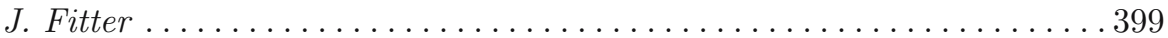

17.1 Introduction ................................... 399

17.1.1 Dynamics in Proteins:

Types of Motions and Their Biological Relevance........ 400

17.2 Samples in Neutron Spectroscopy:

Sample Preparation, Sample Characterization, and Sample Environment ....................... 403

17.3 From Spectra to Results: Data Acquisition, Data Analysis, and Data Interpretation .......................... 405

17.4 Applications and Examples ........................ 412

17.4.1 Comparison of Folded and Unfolded States ........... 412

17.4.2 Conformational Entropy Calculation from Neutron Scattering Data ................................. 415

17.5 Conclusions and Outlook .......................4 416

References ..................................4 417

18 Relating Protein Dynamics to Function and Structure: The Purple Membrane

U. Lehnert, M. Weik ....................................4 419

18.1 Introduction ............................... 419

18.1.1 Elastic Incoherent Neutron Scattering ............. 420

18.2 Methods of Investigation . . . . . . . . . . . . . . . . . . 421

18.2.1 Elastic Incoherent Neutron Scattering on Powder Samples . 421

18.2.2 Models for Describing Thermal Protein Dynamics ........ 421

18.2.3 H/D Labeling Techniques ...................... 423

18.3 Relating Thermal Motions in Purple Membranes

to Structural and Functional Characteristics

of Bacteriorhodopsin .............................4424

18.3.1 Thermal Motions in Bacteriorhodopsin and the Purple

Membrane ..................................4424

18.3.2 Hydration Dependence of Thermal Motions............ . 426

18.3.3 Local Core Motions . . . . . . . . . . . . . . . . . . . . . 427

18.3.4 Lipid Environment ....................... 428

18.3.5 Relation Between PM Dynamics and Crystallographic B-factors .................. 429

18.3.6 Comparison of Force Constants with Forces Measured

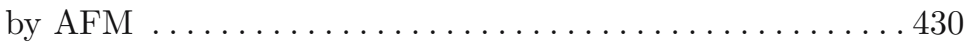

18.4 Protein Dynamics and Function in Some Other Proteins........ . 431

18.5 Conclusions.................................. 432

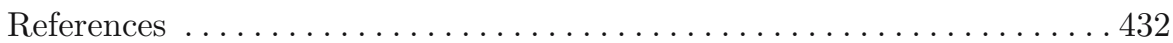




\section{Biomolecular Spectroscopy}

\section{Using Pulsed-Source Instruments}

H.D. Middendorf. ................................. 435

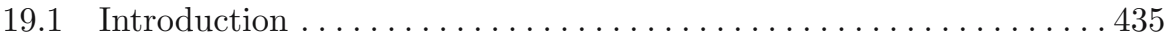

19.2 Why Pulsed Sources? . . . . . . . . . . . . . . . . . . . . . 435

19.3 Pulsed Source vs. Reactor Instruments . . . . . . . . . . . . . . . . . . . . . 437

19.4 Backscattering Spectrometers . . . . . . . . . . . . . . . . . 439

19.4.1 Hydration Dynamics. . . . . . . . . . . . . . . . . . . . . 440

19.4.2 Low-Temperature Dynamics and Glass-Like Transitions . . . 441

19.4.3 Enzyme Dynamics and Folding-Unfolding Processes . . . . . 443

19.5 Inelastic Scattering

at $1 \mathrm{meV}<\hbar \omega<1 \mathrm{eV}\left(8<\hbar \omega<8,000 \mathrm{~cm}^{-1}\right) \ldots \ldots \ldots \ldots \ldots . \ldots 445$

19.5.1 Chopper Spectrometers . . . . . . . . . . . . . . . 446

19.5.2 Crystal-Analyzer and Filter-Difference Spectrometers . . . . 446

19.5.3 Building Blocks and Model Compounds . . . . . . . . . . . . . 449

19.5.4 Interpretational Aspects . . . . . . . . . . . . . . . . . . . . . . . . . 451

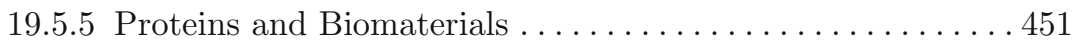

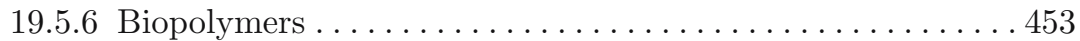

19.5.7 Nucleotides and Nucleosides . . . . . . . . . . . . . . . . . 455

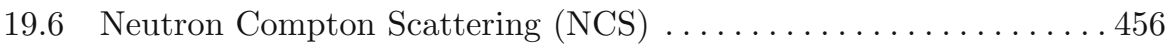

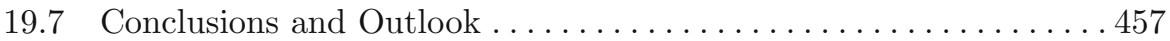

References .................................... 458

\section{Brownian Oscillator Analysis of Molecular}

\section{Motions in Biomolecules}

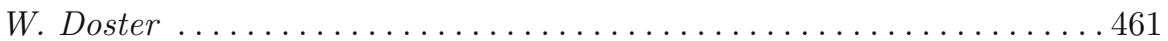

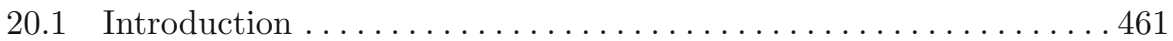

20.2 Dynamics of Protein-Solvent Interactions . . . . . . . . . . . . . . . 461

20.3 Properties of the Intermediate Scattering Function . . . . . . . . . 463

20.4 Relevant Time and Spatial Scales. . . . . . . . . . . . . . . . . . . . . 467

20.5 The Brownian Oscillator as a Model of Protein-Residue Motion . . . . . . . . . . . . . . . . . . . . . . . . . . . 467

20.6 The Visco-Elastic Brownian Oscillator . . . . . . . . . . . . . . . . 470

20.7 Moment Analysis of Hydration Water Displacements . . . . . . . . . . 474

20.8 Analysis of Protein Displacements . . . . . . . . . . . . . . . . . 476

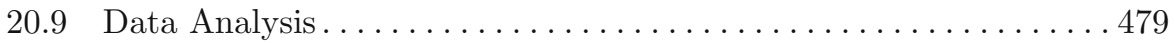

20.10 Conclusions . . . . . . . . . . . . . . . . . . . . . . . . . . . 481

References .................................. 482

21 Internal Dynamics of Proteins and DNA:

Analogy to Glass-Forming Systems

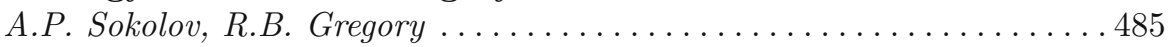

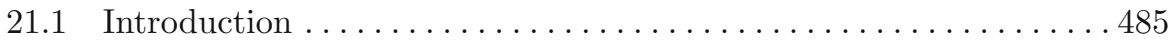

21.2 Analysis of Relaxation Spectra:

Susceptibility Presentation vs. Dynamic Structure Factor . . . . . . 486 
21.3 Slow Relaxation Process. . . . . . . . . . . . . . . . . . . . . . 487

21.4 The Nature of the Dynamical Transition in Proteins and DNA . . 492

21.5 Fast Picosecond Relaxation ....................... 496

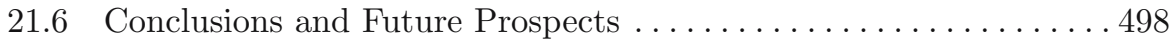

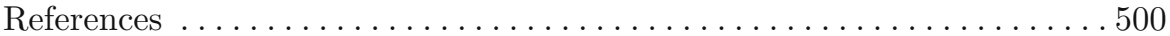

22 Structure and Dynamics of Model Membrane Systems

Probed by Elastic and Inelastic Neutron Scattering

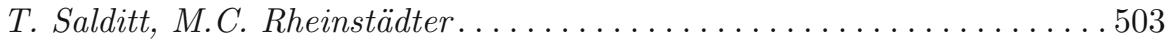

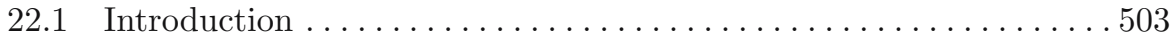

22.2 Sample Preparation and Sample Environment . . . . . . . . . . . . . . 504

22.3 Specular Neutron Reflectivity . . . . . . . . . . . . . . . . . 506

22.4 Nonspecular Neutron Reflectivity . . . . . . . . . . . . . . . . . . . 510

22.4.1 Models of Bilayer Undulations . . . . . . . . . . . . . . . . 512

22.4.2 Monochromatic NSNR Experiments . . . . . . . . . . . . . 513

22.4.3 White-Beam NSNR Experiments . . . . . . . . . . . . . 514

22.4.4 Change of Fluctuations

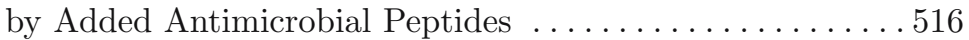

22.5 Elastic and Inelastic Studies

of the Acyl Chain Correlation Peak . . . . . . . . . . . . . . . 518

22.5.1 Inelastic Neutron Scattering . . . . . . . . . . . . . . . . . . 518

22.5.2 Elastic Neutron Scattering . . . . . . . . . . . . . . . . 521

22.5 .3 Collective Dynamics . . . . . . . . . . . . . . . . . . . 523

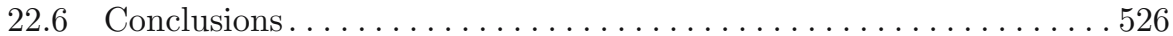

References .................................. 528

23 Subnanosecond Dynamics of Proteins in Solution:

MD Simulations and Inelastic Neutron Scattering

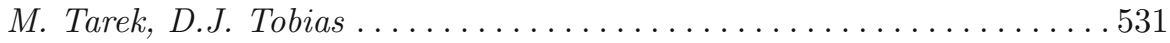

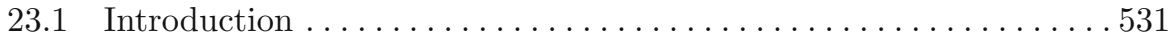

23.2 MD Simulations . . . . . . . . . . . . . . . . . . . . . . . 534

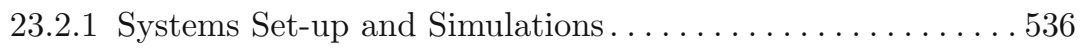

23.2.2 Generating Neutron Spectra . . . . . . . . . . . . . . . . . 537

23.3 Overall Protein Structure and Motion in Solution . . . . . . . . . . 539

23.3.1 Internal Protein Dynamics . . . . . . . . . . . . . . . . 543

23.3.2 Dynamics of Proteins in Solution from MD Simulations . . . 544

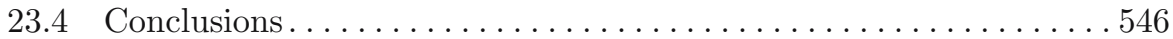

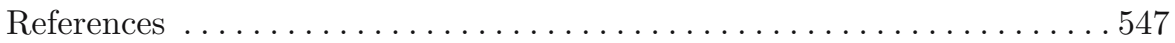

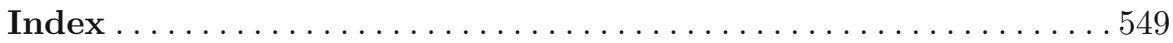

